\title{
Rheumatoid arthritis: from basic findings and clinical manifestations to future therapies
}

\author{
Paul Hasler $^{1} \cdot$ Cem Gabay ${ }^{2}$
}

Received: 19 April 2017 / Accepted: 20 April 2017

(C) Springer-Verlag Berlin Heidelberg 2017

It has been over 10 years since the topic of rheumatoid arthritis (RA) was considered in the pages of Seminars in Immunopathology [1]. At the time, the focus was on the biologics, which had recently entered the stage as mainstream therapies. The improvement in outcome achieved with these agents has been striking, and for most patients with access to biologic therapies, the detrimental effects of RA disease progression have to a large extent become preventable. These include structural deformities of joints with functional limitations, as well as systemic inflammation with premature vascular and other organ diseases with morbidity and early death. The practical success of new therapies with different modes of action has also challenged concepts of pathogenesis of RA [2, 3]. Meeting this challenge has broadened the opportunities for the investigation of basic immunological mechanisms. Both the innate and the adaptive arms of the immune system and the interactions between the many cell types involved in inflammation have yielded important insights into the individual components of inflammation targeting joint tissues. In addition, abundant attention to the interplay between environmental and intrinsic influences has resulted in several new

This article is a contribution to the special issue on Immunopathology of Rheumatoid Arthritis - Guest Editors: Cem Gabay and Paul Hasler

Paul Hasler

paul.hasler@ksa.ch

$\checkmark$ Cem Gabay

Cem.Gabay@hcuge.ch

1 Division of Rheumatology, Cantonal Hospital of Aarau, Aarau, Switzerland

2 Division of Rheumatology, University Hospital of Geneva, Geneva, Switzerland concepts of disease initiation with potential impacts on modes of intervention.

Against this background, we asked ourselves which insights into RA disease mechanisms would be most promising for launching the next breakthroughs in clinical outcomes. In accordance with recent developments, the answer comprises a major broadening of concepts. Findings of genetic and immunopathologic features still show impressive advances. This applies to cross talk between intracellular signaling systems, cell surface responses, and the cellular environment as well as tissue and systemic inflammation. Innate immunity has surprised with a range of novel results, while specific immune responses continue to yield significant findings. Interactions with environmental factors affecting the airways and the gut have gained prominence and impact many facets of genetic, innate, and specific immunity. The limits of opportunities arising from this field are not nearly in sight. A shift to research on human tissue and to clinical and epidemiologic investigation appears to have set in, though animal models retain their value for studies of many disease mechanisms.

Considering this trove of developments, we selected the topics for this issue to highlight the progresses in understanding the basic immunopathology of RA. The article of Douglas Veale, Carl Orr, and Ursula Fearon [4] provides a general overview regarding the interplay between genes and environment in the development of autoimmunity that precedes articular manifestations by many years. They also discuss the immunopathological findings present in the synovial tissue, as well as the role of cytokines and other mediators of inflammation in joint inflammation and tissue damage.

Georg Schett and Ulrike Harre [5] describe the mechanisms responsible for the development of structural damage associated with RA, including bony erosive changes and cartilage breakdown. Regarding bone erosions, they show that in addition to the well-known cellular and molecular mechanisms 
involved in the maturation and activation of osteoclasts, such as the release of RANKL (receptor activator of nuclear factor kappa-B ligand) by synovial fibroblasts, recent data pointed toward a direct effect of anti-citrullinated protein antibodies (ACPA) via their binding and stimulation of pre-osteoclasts. This novel finding provides some rationale regarding the clinical association between the presence of ACPA and an increased risk for the development of erosive RA. In addition, as mentioned by the authors, the anabolic function of osteoblasts is altered in RA leading to decreased new bone formation and impaired repair. This phenomenon is mediated by the inhibitory effect of TNF on Wnt signaling. Various factors at play in joint inflammation are the focus of a detailed review on cytokine signaling by Mélissa Noack and Pierre Miossec [6]. In addition to the well-known effects of TNF, IL-1, and IL-6 on synovial inflammation and joint damage, they discuss the roles of IL-17 and GM-CSF; the latter being currently studied in clinical trials. Asif Amin, David Fox, and Jeffrey Ruth give attention to cellular and molecular markers present in the rheumatoid synovium [7]. In particular, they provide a detailed description of changes that occur in RA synovial fibroblasts leading to cell proliferation, loss of contact inhibition, and activation of inflammatory response via the release of cytokines and chemokines, as well as by cell-cell direct interactions. The proportion of the various immune cells, including lymphoid or myeloid cells, infiltrating the synovial membrane as well as the development of tertiary lymphoid structures is associated with different histopathological patterns. Most importantly, recent data suggest that molecular markers associated with predominant lymphoid or myeloid cell infiltration can be predictive of clinical response to either TNF or IL-6 antagonists.

Gene expression remains a foundation for recognizing individual predisposition to develop RA and for studying its immunopathology. Sebastien Viatte and Anne Barton discuss the current understanding of the hereditary contribution, primarily by HLA alleles, with consideration of the insights gleaned from genome wide association studies and the implications for recognizing disease susceptibility and response to therapy [8]. Layered on the genetic code are manifold epigenetic alterations that influence gene expression and carry over into the contribution of innate and adaptive immunity to inflammation in RA, as Caroline Ospelt, Steffen Gay, and Kerstin Klein explain [9].

Alexander Tracy, Christopher Buckley, and Karim Raza summarize the evidence provided by studies on banked serum that have revealed developing autoantibody production and immune cell alterations long before disease manifestations become apparent [10]. They describe the multiple factors that influence the development of autoimmunity and present data that support the concept that the transitions from genetically atrisk states to establish RA depend on multiple "switches". This offers the opportunity for implementing screening and preemptive measures, making a vital issue of early recognition of at risk individuals and the likely disease course for optimal prevention and management. Veerle Derksen, Tom Huizinga, and Diane van der Woude follow up on this topic with the many facets playing into the generation of autoantibodies, the roles the antibodies may play in the pathology of disease, and potential points of intervention [11].

Despite the emphasis on arthritis, the systemic effects of ongoing inflammation in RA have been a main concern for decades. Kim Lauper and Cem Gabay [12] comment the basic and clinical evidence gained since the problem of cardiovascular morbidity and mortality in RA was ascertained [13]. Chronic inflammation, in addition to traditional risk factors, is a key player in cardiovascular events via activation of endothelial cells, infiltration of immune cells within arterial walls, and fragmentation of atherosclerotic plaques. Inflammation induces also marked changes of the lipid profile, including decreased cholesterol levels and modification of the protein content of HDL particles. Current recommendations on how to manage cardiovascular risk in patient care are reviewed.

Advances in linking environmental factors to disease processes have identified an assortment of potential initiating elements, from the inhalation of tobacco smoke, other airborne pollutants to infectious disease. In the last few years, the composition of the gut microbiome has garnered increasing attention regarding its potential to regulate not only metabolic homeostasis, but also to sustain appropriate immune system activity. Alterations in the microbiome appear to disturb metabolism and reactivity of the immune system, the latter with links to increased inflammatory responsiveness. Deshiré Alpízar-Rodríguez and Axel Finckh answer the fascinating questions arising from these findings [14].

Though animal models of RA may have limited applicability in the human setting, the tasks of investigating the manifold interactions merging into the inflammatory phenotype of RA justify their judicious application to manipulate individual effector components of inflammation with the potential to destroy joint structure. These are issues addressed by Natacha Bessis and colleagues [15].

Tommy Cheung and Iain McInnes deliberate the ultimate aim of extending the knowledge on RA, the introduction of ever more effective measures against the articular and systemic inflammation, while avoiding undue compromises of immune defense and other undesired effects [16]. They give particular attention to therapies, including GM-CSF and JAK inhibitors that are currently in advanced stage of clinical development, as well as other targeted therapies that are investigated in pre-clinical models or that are still in early clinical development, including Bruton's tyrosine kinase and phosphoinositide-3-kinase inhibitors, autonomous nervous system modulators, and cell therapy with the use of "tolerogenic" dendritic cells. 
The fundamental insights provided by the contributors to this issue, whether derived from basic or from clinical and epidemiologic investigations, broaden the scope for interventions and further advances in knowledge. These pertain to the prevention of disease with tools to modify the environment, or the immune responses to the environment, even before disease becomes apparent. The introduction of promising novel drugs with improved efficacy and fewer side effects appears imminent. They may be combinable in original ways to improve disease in patients hitherto unresponsive to therapy. In addition, nonpharmaceutical measures such as modifying the microbiota and the metabolism of immune system components may be effective at various stages of disease. We are excited by these prospects and look forward intensely to the developments of the coming years. Will they be as groundbreaking as the introduction of the biologics? Even if we do not know where the answer lies, the prospects should motivate an all-out effort to surpass past achievements in the next few years!

Finally, we would like to cordially thank all the authors for seizing the opportunity to supply the reviews that make up this issue of Seminars in Immunopathology. They represent the cutting edge of current knowledge, with its encouraging outlook for a continued intense pace of progress. Many more exciting and practically applicable findings may perhaps even achieve the ultimate goals of preventing and curing RA.

\section{References}

1. Hasler P (2006) Biological immunomodulators. Springer Semin Immunopathol 27(4):389-390. doi:10.1007/s00281-006-0017-4

2. McInnes IB, Schett G (2011) The pathogenesis of rheumatoid arthritis. N Engl J Med 365(23):2205-2219. doi:10.1056/ NEJMra1004965
3. Smolen JS, Aletaha D, McInnes IB (2016) Rheumatoid arthritis. Lancet (London, England) 388(10055):2023-2038. doi:10.1016/ s0140-6736(16)30173-8

4. Veale DJ, Orr C, Fearon U (2017) Cellular and molecular perspectives in rheumatoid arthritis. Semin Immunopathol. doi:10.1007/ s00281-017-0633-1

5. Schett G, Harre U (2017) Cellular and molecular pathways of structural damage in rheumatoid arthritis. Semin Immunopathol. doi:10. 1007/s00281-017-0634-0

6. Noack M, Miossec P (2017) Selected cytokine pathways in rheumatoid arthritis. Semin Immunopathol. doi:10.1007/s00281-0170619-z

7. Amin MA, Fox DA, Ruth JH (2017) Synovial cellular and molecular markers in rheumatoid arthritis. Semin Immunopathol. doi:10. 1007/s00281-017-0631-3

8. Viatte S, Barton A (2017) Genetics of rheumatoid arthritis susceptibility, severity and treatment response. Semin Immunopathol. doi: 10.1007/s00281-017-0630-4

9. Ospelt C, Gay S, Klein K (2017) Epigenetics in the pathogenesis of RA. Semin Immunopathol. doi:10.1007/s00281-017-0621-5

10. Tracy A, Buckley CD, Raza K (2017) Pre-symptomatic autoimmunity in rheumatoid arthritis: when does the disease start? Semin Immunopathol. doi:10.1007/s00281-017-0620-6

11. Derksen VFAM, Huizinga TWJ, van der Woude D (2017) The role of autoantibodies in the pathophysiology of rheumatoid arthritis. Semin Immunopathol. doi:10.1007/s00281-017-0627-z

12. Lauper K, Gabay C (2017) Cardiovascular risk in patients with rheumatoid arthritis. Semin Immunopathol. doi:10.1007/s00281017-0632-2

13. Pincus T, Callahan LF (1990) Remodeling the pyramid or remodeling the paradigms concerning rheumatoid arthritis-lessons from Hodgkin's disease and coronary artery disease. J Rheumatol 17(12): $1582-1585$

14. Alpízar-Rodríguez D, Finckh A (2017) Environmental factors and hormones in the development of rheumatoid arthritis. Semin Immunopathol. doi:10.1007/s00281-017-0624-2

15. Bessis N, Decker P, Assier E, Semerano L, Boissier MC (2017) Arthritis models: usefulness and interpretation. Semin Immunopathol. doi:10.1007/s00281-017-0622-4

16. Cheung TT, McInnes IB (2017) Future therapeutic targets in rheumatoid arthritis? Semin Immunopathol. doi:10.1007/s00281-0170623-3 